\title{
Morphofunctional, viability and antioxidant system alterations on rat primary testicular cells exposed to simulated microgravity
}

\author{
Valentina Bonetto, ${ }^{1}$ Linda Scarabelli, ${ }^{2}$ Maria Angela Masini ${ }^{1}$ \\ ${ }^{1}$ DISIT, University of Eastern Piedmont, Alessandria; ${ }^{2}$ DIMES, University of Genoa, Genova, Italy
}

\begin{abstract}
This study focused on effects induced by Short-term Simulated Microgravity (SMG) condition on primary cell culture from pre-pubertal Wistar rats testis. Cells were analyzed for cytoskeletal and Sex Hormone Binding Globulin (SHBG/ABP) changes by immunofluorescence technique, for antioxidant system exploiting RT-PCR and cell viability. Cells were cultured for 6 and $24 \mathrm{~h}$ on a three-dimensional clinostat, Random Positioning Machine (RPM). At the end of each experiment, once stopped the RPM rotation, cells were either fixed in paraformaldehyde or lysed and RNA extracted. In cells exposed to SMG the cytoskeleton became disorganized, microtubules fragmented and SHBG was already undetectable after $6 \mathrm{~h}$ of treatment. Moreover, various antioxidant systems significantly increased after $24 \mathrm{~h}$ of SMG exposure. Initially, SMG seemed to disturb antioxidant protection strategies allowing the testes to support sperm production, thus generating an aging-like state of oxidative stress. Studies on changes induced by short-term altered gravity conditions, carried out in real microgravity, could give more information on steroido-
\end{abstract}

Correspondence: Valentina Bonetto, DISIT, University of Eastern Piedmont, Via T. Michel 11, 15121 Alessandria, Italy.

Tel.: +39.0131.360274 Fax: +39.0131.360390

E-mail: valentina.bonetto@uniupo.it

Key words: Testicular primary culture; simulated microgravity; cytoskeleton; oxidative stress.

Acknowledgements: This work was supported by the Italian Space Agency (ASI).

Contributions: VB: histology, immunohistochemistry, mitocapture, data analysis; LS: PCR; MAM: design of the research, data analysis.

Conflict of interest: The authors have no conflict of interest to declare.

Received for publication: 24 May 2021.

Revision received: 9 October 2021.

Accepted for publication: 16 October 2021.

${ }^{\circ}$ Copyright: the Author(s), 2021

Licensee PAGEPress, Italy

Journal of Biological Research 2021; 94:9875

doi:10.4081/jbr.2021.9875

This article is distributed under the terms of the Creative Commons Attribution Noncommercial License (by-nc 4.0) which permits any noncommercial use, distribution, and reproduction in any medium, provided the original author(s) and source are credited. genesis and germ cell differentiation within the testis exposed to this condition and confirm the validity of simulation approach.

\section{Introduction}

Since the advent of space flights more than 530 men and women have travelled to space, introducing the problem concerning the effects of microgravity on human physiology. ${ }^{1}$ Nowadays it is well known that any alteration of the gravitational force causes relevant physiological changes in organisms, and it may affect their reproductive physiology and fertility. ${ }^{2-4}$ During spaceflight, the astronauts experiment not only microgravity, but also hypergravity, which occurs during launch and re-entry phases, that is another interesting topic investigated by several researchers. ${ }^{2,5,6}$

In the present study, testis has been chosen because of its relevance as an endocrine organ and because of its key role in the reproductive system. The investigation of reproductive health requires extensive studies using animals and cell culture models, in order to fill gaps regarding the effect of long duration space missions on mammalian reproduction, starting from changes induced by short-term altered gravity conditions.

Due to the limited possibilities to perform experiments in real microgravity, several methods have been developed to Simulate Microgravity (SMG) such as the randomization of the direction of gravity force using a 3D-clinostat, as Random Positioning Machine (RPM). ${ }^{2,7}$ Studies on the effect of weightlessness on the reproductive system of rodent models, conducted in real and Simulated Microgravity (SMG), showed similar morpho-functional testicular alterations. ${ }^{8,9}$ The results obtained in rodent studies and experiments, made by others researchers and our group, showed an increase of physiological and morphological changes on reproductive system using different gravity vectors $(0 \mathrm{~g}$, through $1 \mathrm{~g}$ to $2 \mathrm{~g}) .{ }^{10-12}$ Moreover, such findings are consistent with the results of cell biology studies using SMG, which show morphological and functional changes to occur in testicular cells exposed to RPM. More in detail, these studies demonstrates that SMG causes changes in the seminiferous tubules, with altered tubular architecture and reduction in the number of spermatogenic cells, in addition microgravity induce hypogonadism directly rather than through stress-induced gonadal inhibition. ${ }^{12}$ On the other hand, rats under SMG showing variation in testosterone levels demonstrated complete protection of both muscle and bone using a combination of bisphosphonate and testosterone, suggesting that androgen deficiency contributes at least partially to the pathophysiology of space flight-related muscle and bone atrophy. ${ }^{10}$ As regard to humans, a decrease in testosterone secretion was also observed in astronauts during space flights. ${ }^{13}$ 
At cellular level, both real and simulated microgravity has proved to be one of the stress environmental factors that results in severe damages to the cytoskeleton of cells kept in culture. ${ }^{14-16}$ Results of different studies shown damages to the cytoskeleton of Sertoli cell line and the lymphocytes' cytoskeleton both during spaceflight and in SMG. ${ }^{12,14,17,18}$ Spermatogonial cell differentiation into mature spermatozoa requires Sertoli cells, which play a crucial role in the development of germ cells and in the regulation of spermatogenesis. In addition to its nursing function, Sertoli cells produce a large number of proteins essential for germ cell survival and development. ${ }^{12}$

The aim of this study was to evaluate the influence on a primary cell culture from rat testis, at first stages of exposure to SMG; cells were exposed for 6 and $24 \mathrm{~h}$ to the treatment and analyzed by immunohistochemistry, molecular biology and general chemistry techniques, in order to evaluate the cell functionality after short-term SMG treatment. The intervals of time were chosen according to previous works that demonstrate alterations in cellular structure and functionality already after $6 \mathrm{~h}$ and important changes at 24 h. ${ }^{19,20,21}$

\section{Materials and Methods}

\section{Preparation of cell culture}

Cells were isolated from pre-pubertal ( 8 days old) Wistar rats and grown in DMEM (Sigma, St. Louis, Missouri) with the addition of $10 \%$ Fetal Bovine Serum, $1 \%$ L-glutamine, $1 \%$ gentamicin, streptamicin and amphotericin, at $34^{\circ} \mathrm{C}$ in a $5 \% \mathrm{CO}_{2}$ incubator. The cells were seeded at 20,000 cells $/ \mathrm{mL}$ using "flasks on a slide" (flasks located onto a removable slide, $9.0 \mathrm{~cm}^{2}$, Thermo Scientific Nunc). The flasks were positioned as close as possible to the center of the platform on the RPM (Dutch Space, Leiden, The Netherlands) and kept under rotation at $56 \mathrm{deg} / \mathrm{sec}$, using the real random mode of the instrument, for $6 \mathrm{~h}$ and $24 \mathrm{~h}\left(\mathrm{SMG}, 10^{-6} \mathrm{~g}\right.$ ) (Figure 1). Static controls (ground controls, $1 \mathrm{~g}$ ), were cultured for $24 \mathrm{~h}$ under normal gravity conditions onto the supporting frame of the instrument in order to get cells to the same vibration stress conditions.

At the end of the experiment, once stopped the RPM rotation, some flasks were fixed with Phosphate Buffered Saline (PBS) containing $4 \%$ paraformaldehyde. Fixed cells were submitted to immunohistochemical techniques and to detect apoptotic cells with Mitocapture Mitochondrial Apoptosis detection kit
(Biovision, Milpitas, CA), whereas other flasks were used to obtain cells for RT-PCR.

Microgravity conditions were simulated using an RPM connected to a control console through standard electrical cables. The apparatus is a $3 \mathrm{D}$ clinostat consisting of two independently rotating frames. One frame is positioned inside the other, exerting a complex net change in orientation on a biological sample mounted in the center of the RPM platform. ${ }^{22}$ The rotation of the sample induces centripetal acceleration but placing the sample in the center of the RPM platform this effect will be negligible. Moreover, culture flasks were completely filled with medium without gas bubbles, avoiding loss of liquid during rotation. Gas bubbles result in unwanted fluid motion and associated shear stress to the sample. ${ }^{22}$ This device does not actually eliminate gravity, but the RPM is a micro-weight simulator based on the principle of "gravity-vector averaging": it allows a $1 \mathrm{~g}$ stimulus to be applied omnidirectionally, and the sum of the gravitational force vectors tends to equal zero. The effects generated by the RPM are comparable to the effects of real microgravity, provided that the direction changes are faster than the response time of the system to the gravity field. The desktop RPM used was positioned within an incubator set at $34^{\circ} \mathrm{C}$, value close to the physiological testicular temperature, and $5 \% \mathrm{CO}_{2}$ to match physiologic conditions and to maintain a constant $\mathrm{pH} .^{22}$

\section{Immunohistochemical techniques}

The slides removed from the flasks, containing the cell cultures, were submitted to the indirect immunofluorescence technique. $^{23}$ After permeabilization with Triton X-100 (Sigma, St. Louis, Missouri) $01 \%$ in phosphate buffered saline (PBS, $0.01 \mathrm{M}$, $\mathrm{pH}$ 7.4), PBS washing and exposure to Normal Goat Serum (diluted 1:50 in PBS; Sigma, St. Louis, Missouri) in a moist chamber at $20^{\circ} \mathrm{C}$, the cells were incubated overnight at $4^{\circ} \mathrm{C}$ with the antisera to $\alpha$-tubulin (raised in mouse, diluted 1:500 in PBS, Sigma, St. Louis, Missouri) or to Sex Hormones Binding Globulin (SHBG/ABP, raised in rabbit against aminoacids 197-403 mapping at the C-terminus of SHBG of mouse origin, diluted 1:100, Santa Cruz Biotechnology, Inc). After PBS washing, a second layer of fluoresceine-isothiocyanate conjugated $\gamma$-globulins (FITC), goat anti-mouse (diluted 1:100 in PBS, Sigma, St. Louis, Missouri) and goat anti-rabbit (diluted 1:100 in PBS, Sigma, St. Louis, Missouri) following the specificity of the antisera for $30 \mathrm{~min}$ into a moist chamber, at $20^{\circ} \mathrm{C}$. The slides were rinsed in PBS, mounted with gel-mount (Biomeda Corp., Foster City, CA). The specificity of the immunostainings was verified by omitting one of the steps of the

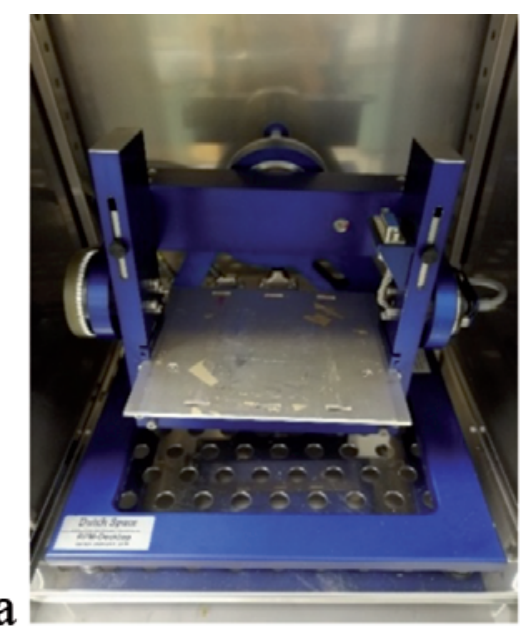

a

b

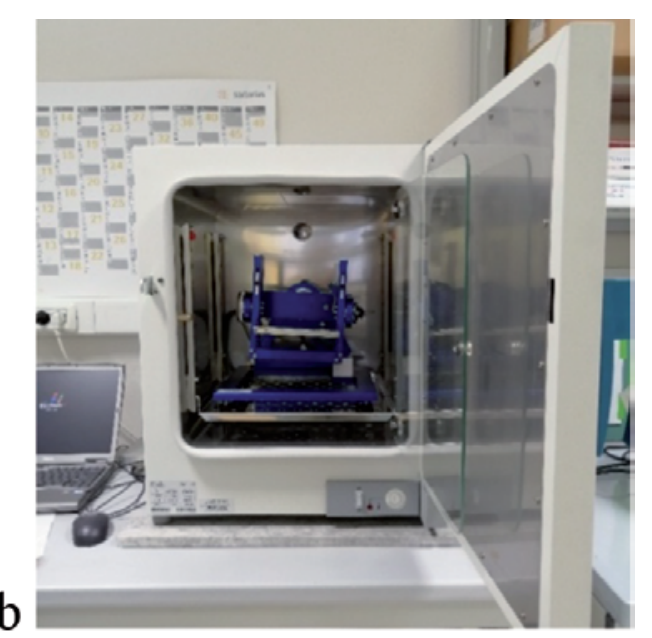

Figure 1. Random Positioning Machine (RPM).The RPM (Dutch Space, Leiden, Netherlands) (a) was run in a commercially available incubator at $34^{\circ} \mathrm{C}$ and $5 \% \mathrm{CO}_{2}(\mathrm{~b})$. 
immunohistochemical procedure, or by replacing the primary antisera with non-immune rabbit serum or PBS. Immunoreactions were visualized using a conventional epifluorescence microscope (Leica Axiovert).

\section{RNA isolation and real-time RT-PCR}

Since cytoskeleton alterations, we investigated the level of oxidative stress after $24 \mathrm{~h}$ of SMG by measuring the expression level of genes involved in ROS metabolism or antioxidant defense mechanism. Total RNA was isolated by the acid phenolchloroform procedure using the Trizol reagent (Sigma, St. Louis, Missouri) according to the manufacturers' instructions. ${ }^{24}$ The purity of RNA was checked via absorption spectroscopy by measuring the $260 / 280$ ratio. Only high purity samples $\left(\mathrm{OD}_{260} / 280>1.8\right)$ were subjected to further manipulation. The quality of isolated RNA was assessed by electrophoresis on $1.5 \%$ formaldehyde-agarose gel to verify the integrity of the $18 \mathrm{~S}$ and $28 \mathrm{~S}$ rRNA bands. First strand cDNA was synthesized from $1 \mu \mathrm{g}$ of total RNA using 200 ng oligo(dT)18-primer (TIB MolBiol, Italia), 200 units RevertAid H-Minus M-MuLV reverse transcriptase (Fermentas, Hannover MD, USA), 40 units RNAsin and 1 $\mathrm{mM}$ dNTPs (Promega, Milan, Italy) in a final volume of $20 \mu \mathrm{L}$. The reaction was performed in a Master-cycler apparatus (Eppendorf, Milan Italy) at $42^{\circ} \mathrm{C}$ for $1 \mathrm{~h}$ after an initial denaturation step at $70{ }^{\circ} \mathrm{C}$ for $5 \mathrm{~min}$. The expression levels of genes were quantified in $96-$-well optical reaction by using a Chromo $4^{\mathrm{TM}}$ System real-time PCR apparatus (Biorad, Milan, Italy). Realtime PCR reactions were performed in quadruplicate in a final volume of $20 \mu \mathrm{L}$ containing $10 \mathrm{ng}$ cDNA, $10 \mu \mathrm{L}$ of iTaq SYBR Green Supermix with ROX (Biorad, Milan, Italy), and $0.25 \mu \mathrm{M}$ of each primer pair (TibMolBiol, Genoa, Italy). The glyceraldehyde 3-phosphate dehydrogenase (GAPDH) was used as a housekeeping gene to normalize the expression data. The accession numbers of the genes used in the study and the primer sequences are given in Table 1 . The thermal protocol included an enzymatic activation step at $95^{\circ} \mathrm{C}(3 \mathrm{~min})$ and 40 cycles of $95^{\circ} \mathrm{C}(15 \mathrm{~s}), 60^{\circ} \mathrm{C}$ (30s) and $72^{\circ} \mathrm{C}(20 \mathrm{~s})$. The melting curve of the PCR products (55-94 ${ }^{\circ} \mathrm{C}$ ) was also recorded to check the reaction specificity. The relative gene expression of target genes in comparison of the GAPDH reference gene was conducted following the comparative $\mathrm{C}_{\mathrm{T}}$ threshold method using the Biorad software tool GenexGene Expression Macro ${ }^{\mathrm{TM}}{ }^{25,26}$ The normalized expression was then expressed as the relative quantity of mRNA (fold induction) with respect to the control sample. Data are the mean $\pm \mathrm{SD}$ for three experiments in quadruplicate.

\section{Cell viability assays}

Cell death was assessed by staining with MitoCapture (Biovision, Milpitas, CA), a fluorescent lipophilic cationic reagent that assesses mitochondrial membrane permeability, according to the supplier's instructions. Briefly, cells were incubated with the MitoCapture reagent for $15 \mathrm{~min}$ at $37^{\circ} \mathrm{C}$ and observed by fluorescence microscopy using a wide band pass filter. Cells with intact mitochondria exhibited focal red cytosolic fluorescence, whereas cells with permeabilized mitochondria exhibited diffuse green cytosolic fluorescence. Cells lacking red fluorescence and having green fluorescence were scored positive. Cell death was evaluated in each experiment from control and treated cultures. Data were expressed as Integrated Density and the corrected total cell fluorescence (CTCF) calculated from three fields chosen at random in two slide preparations for each sample.

\section{Statistical analysis}

Statistical analysis was performed by using ANOVA followed by Bonferroni ad hoc post test, values were expressed as mean \pm standard deviation (SD; INSTAT software,GraphPad Software, Inc., San Diego, CA 92130 USA).

\section{Results}

\section{Effect of modeled microgravity on cytoskeleton and Sex Hormone Binding Globulin}

After $6 \mathrm{~h}$ in modeled microgravity (Figure 2b), cells didn't show relevant morphological differences as compared with the cells maintained at $1 \mathrm{~g}$ (Figure 2a). The cytoskeleton, identified with a specific antibody directed against $\alpha$-tubulin, was well organized, with microtubules radiating in discrete filaments from the nucleus to the plasma membrane. When the rotation was prolonged to $24 \mathrm{~h}$, the microtubular array was more disorganized and microtubules appeared fragmented; as a consequence, the cells lost their shape after $24 \mathrm{~h}$ of SMG (Figure 2c).

An immunoreactive signal for Sex Hormone Binding Globulin (SHBG/ABP) was detected in the control cells, whereas is totally absent in cells exposed to SMG (Figure 3).

\section{Cell viability assays: MitoCapture}

In healthy cells, the MitoCapture reagent remains in the mitochondria in form of polymers and gives off a red fluorescence; however, in case of mitochondrial damage, the reagent leaks out into cytoplasm as monomers that generate a green signal. As

Table 1. Name and accession numbers of the target genes are listed together with the primer sequences used for RT-PCR analysis in the study.

\begin{tabular}{llll} 
Gene name & Accession number & Forward primer $\left(5^{\prime}-3^{\prime}\right)$ & Reverse primer $\left(3^{\prime}-5^{\prime}\right)$ \\
MT-1 & NM_013602 & CTGCTCCACCGGCGG & GCCCTGGGCACATTTGG \\
MT-2 & NM_008630 & TCCTGTGCCACAGATGGATC & GTCCGAAGCCTCTTTGCAGA \\
\hline p53 & NM_030989 & GGCTCCTCCCCAACATCTTATC & TACCACCACGCTGTGCCGAAAA \\
PARP-1 & NM_013063 & TGCAGTCACCCATGTTCGATGG & AGAGGAGGCTAAAGCCTTG \\
GST & NM_013541 & GTGCCCGGCCAAGAT & TTGATGGGACGGTTCACATG \\
CAT & NM_009804 & CCTGAGAGAGTGGTACATGC & CACTGCAAACCCACGAGGG \\
\hline Mn-SOD & NM_013671 & GGCTCCCGGCACAACACAGCC & CCTCGTGGTACTTCTCCTCGGTG \\
GAPDH & NM_008084 & GACCCCTTCATTGACCTCAAC & CGCTCCTGGAAGATGGTGATGGG \\
\hline
\end{tabular}


demonstrated in Figure 4 the red fluorescence in the control cells (Figure 3a) was replaced by green fluorescence under SMG treatment. After $6 \mathrm{~h}$ (Figure $4 \mathrm{~b}$ ) and $24 \mathrm{~h}$ treatments (Figure $4 \mathrm{c}$ ) the red signal decreased and there is a significant difference in CTCF value related to the red signal in samples cultured under SMG (Figure 4d). This indicates that increasing the time of exposure to SMG increases mitochondrial transmembrane permeability.

a

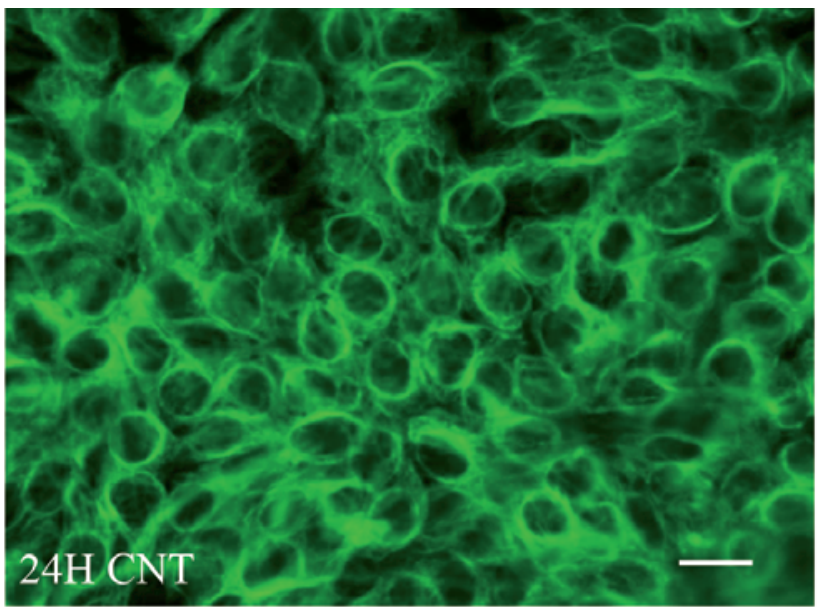

b

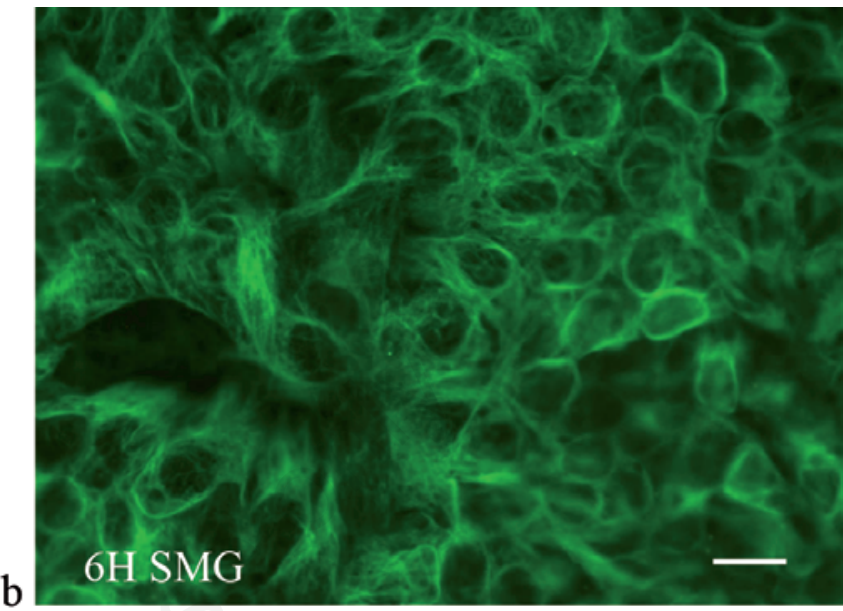

Figure 2. Immunofluorescence analysis of microtubules (antisera to $\alpha$-tubulin, green) of testis primary cell culture cultured at $1 \mathrm{~g}$ or on RPM. Representative immunofluorescence staining for microtubules. Control cells (CNT) cultured for $24 \mathrm{~h}$ in normal gravity condition (a) showed a well organized distribution of tubulin, while in cells exposed to SMG (simulated microgravity) microtubules are disorganized $-6 \mathrm{~h}$ (b) and $24 \mathrm{~h}$ (c). Scale bar: $10 \mu \mathrm{m}$. All images were obtained with the same magnification. c

\section{$24 \mathrm{H} \mathrm{SMG}$}

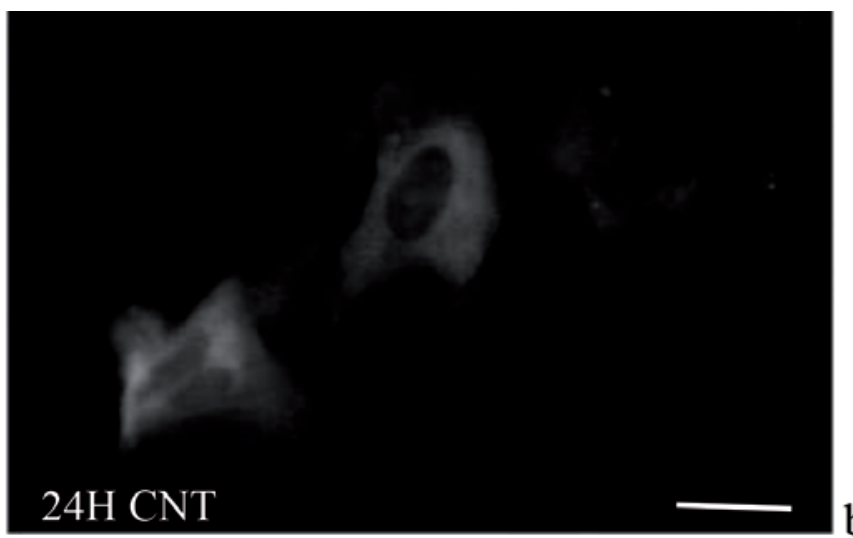

Figure 3. Immunofluorescence analysis for Sex Hormone Binding Globulin/Androgen-Binding Protein (SHBG/ABP). Representative immunofluorescence staining for SHBG/ABP detected in the control cells (CNT) growth for $24 \mathrm{~h}$ in normal gravity condition (a). In cells exposed for $24 \mathrm{~h}$ to simulated microgravity (SMG) the immunostaining is very weak (b). Scale bar: $10 \mu \mathrm{m}$. 
the expression of poly [ADP-ribose] polymerase 1 (PARP-1), an enzyme normally activated by oxidative stress, and the protective role of the oncosuppressor gene p53 were also evaluated. After 24 $\mathrm{h}$ of rotation the expression of MT-1, p53, PARP and GST showed a significant increase with respect to controls (Figure 5).

\section{Discussion}

Colonization of deep space poses a lot of hazards to human reproduction, one of these is the exposure to weightlessness. Experiments carried out with different organisms exposed to microgravity and space flight conditions showed subtle abnormalities in fertilization. Only a few studies concerned mammals, making difficult to fill gaps in knowledge regarding the effect of space mission on mammalian reproduction. ${ }^{12}$

Spermatogenesis is a complex, highly ordered process of cell division and differentiation by which spermatogonia become mature spermatozoa. The understanding of this process in microgravity condition needs to be investigated starting from firsts responses of testes system, in order to evaluate the effects on male fertility. Spermatogenesis depends from intratesticular and extratesticular hormonal regulatory processes and functions of the inter- tubular microvasculature, the Leydig cells and other cellular components of the intertubular space. These signals are integrated, thus allowing the secretion of products that control germ cell development and modulate the function of the other testicular cells, including their own. ${ }^{9}$

After $6 \mathrm{~h}$ in modeled microgravity testis cells (obtained from pre-pubertal 8 days old Wistar rat) showed firsts signs of cytoskeleton alterations, as reported in previous studies and in other cell types (glial, endothelial, thyroid cells or lymphocytes), and that increases over time. ${ }^{14-16,18-20}$ Moreover, gravity vector variation influenced the androgen binding protein (SHBG/ABP) expression, absent in treated testis cells. Considering the role of SHBG/ABP as androgen carrier, the absence of this protein, at $6 \mathrm{~h}$ and $24 \mathrm{~h}$, could significantly compromise the efficiency of spermatogenesis.

During spaceflight most of physiological changes are related to microgravity, ${ }^{1,3,4}$ additionally this condition has been reported to cause cellular oxidative stress that leads to the production of Reactive Oxygen Species (ROS) linked to cellular senescence. ${ }^{27}$ However, the study of microgravity induced oxidative stress is not yet completely understood.

The testes have developed a sophisticated array of antioxidant systems which includes both enzymatic and non-enzymatic constituents. ${ }^{28}$ Our results showed a significant up-regulation of glu- a
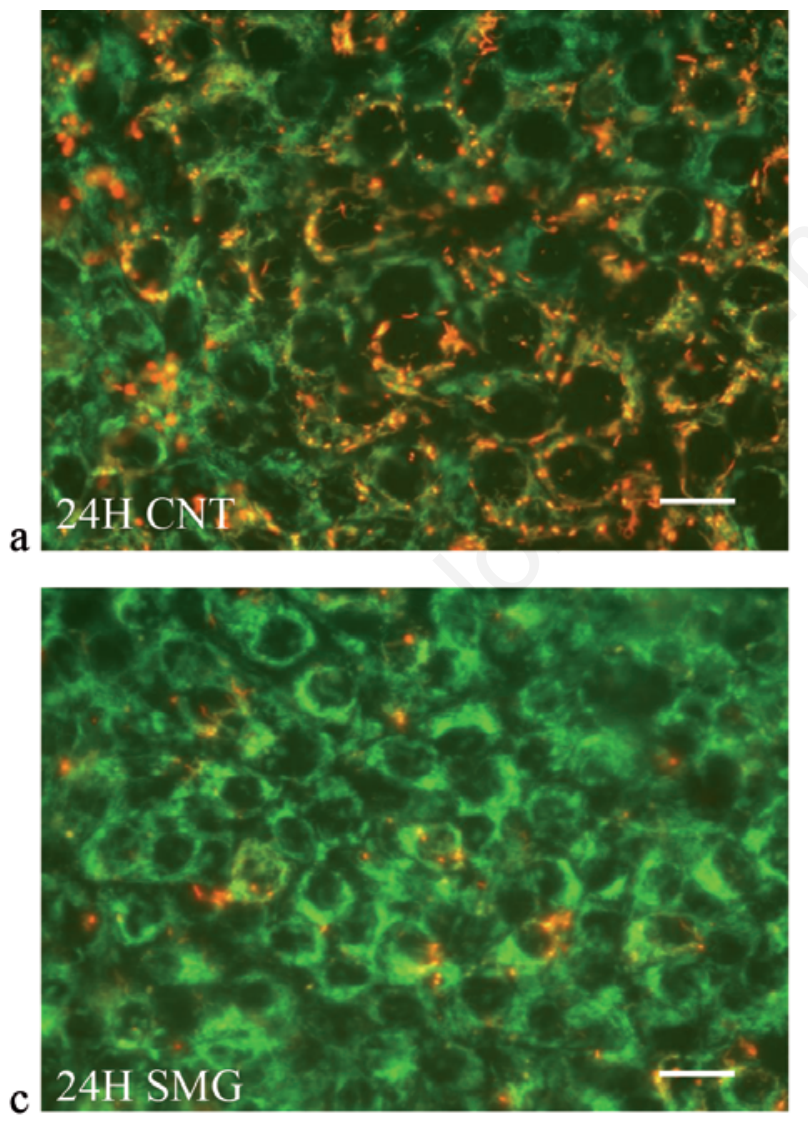
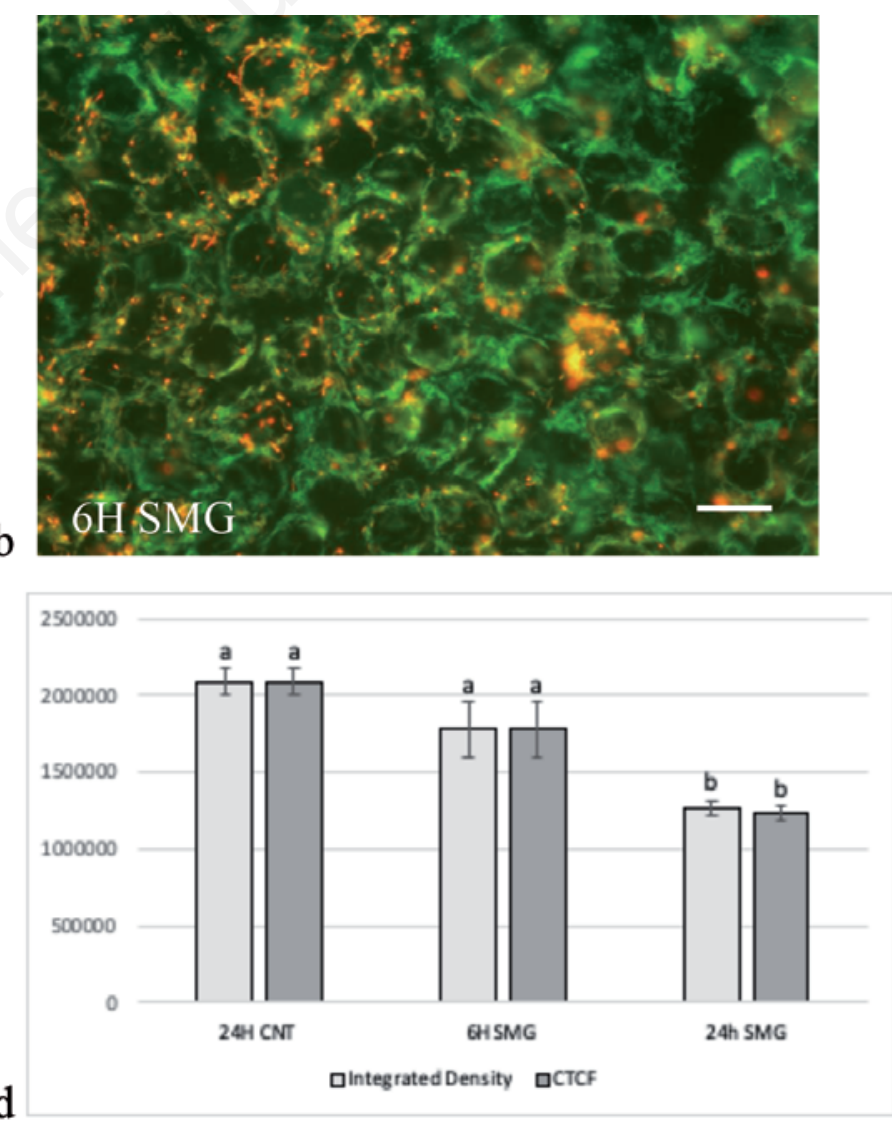

Figure 4. Analysis of cell viability and mitochondrial integrity. Cells were incubated with the MitoCapture reagent to detect mitochondrial membrane integrity after microgravity treatment. The figures show representative images of cells with intact (punctate red fluorescence) or permeabilized (diffuse green fluorescence) mitochondria. Control cells (CNT) cultured for $24 \mathrm{~h}$ in normal gravity condition (a); cells after 6h under SMG (b); cells after $24 \mathrm{~h}$ under SMG (c). Integrated Density and correct total cell fluorescence (CTCF) related to red signal (healthy cells) graph (d). Differences among means were assessed using ANOVA test followed by Bonferroni ad hoc post test. $\mathrm{p} \leq \mathbf{0 . 0 5}$ was considered statistically significant. Scale bar: $10 \mu \mathrm{m}$. All images were obtained with the same magnification. 


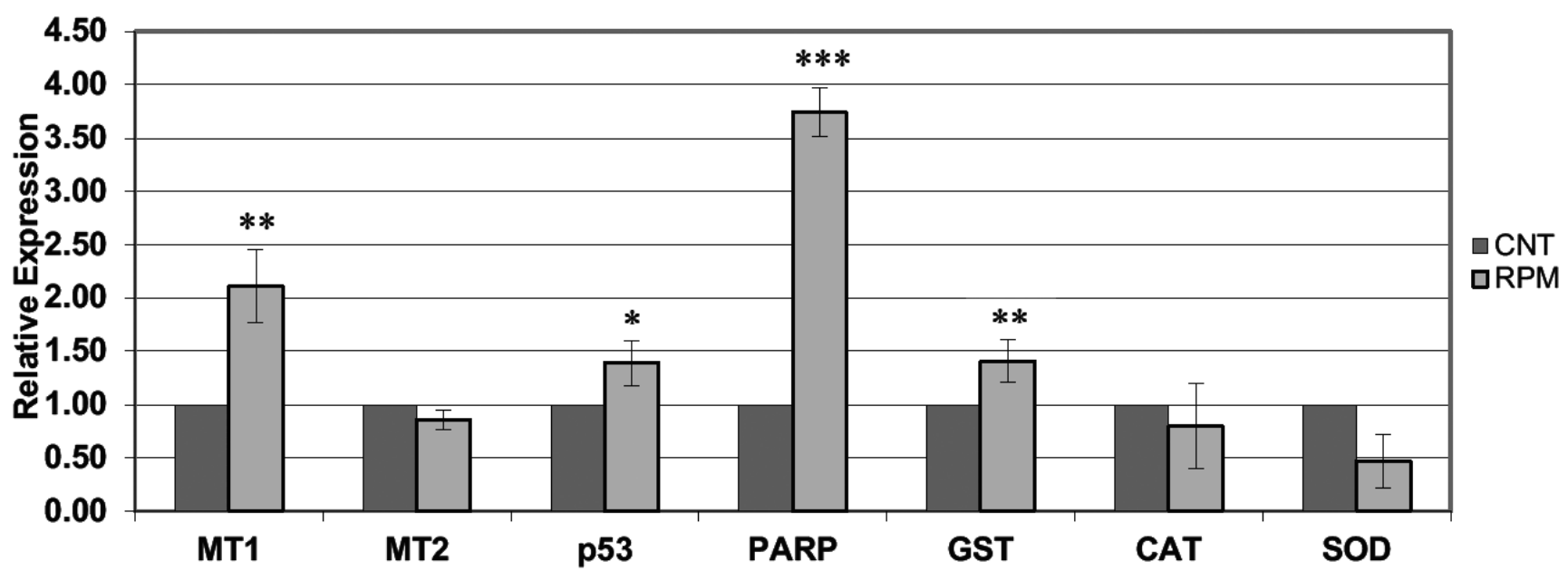

Figure 5. Effects of simulated microgravity on the expression of antioxidant enzymes, p53 and PARP-1 by testis primary cell culture. Relative expression of Metallothioneins (MT-1 and MT-2), p53, PARP-1, Glutathione Transferase (GST), Catalase (CAT) and Superoxide Dismutase (SOD) were quantified in testis cells after $24 \mathrm{~h}$ of Simulated Microgravity (SMG) by real-time RT-PCR. Values represent the fold induction as compared to the Respective Controls (CNT) after normalization for GA3PDH mRNA, used as reference gene. Data are expressed as means \pm SD of three independent experiments. Differences among means were assessed using ANOVA test followed by Bonferroni ad hoc post test. ${ }^{* * *} \mathrm{p}<0.0001,{ }^{* *} \mathrm{p}<0.001,{ }^{*} \mathrm{p}<0.01$ as compared to the respective controls.

tathione S-transferase, GST, active in oxidative and xenobiotic response, that support the hypothesis of a ROS increase at intracellular levels after 24h of SMG. Moreover, higher MT-1 expression level observed could reflect a defensive response or adaptation to the microgravity stress, suggesting an interaction with ROS scavenging enzymes, which agrees with the fundamental role of MTs as maintainers of zinc pool, restoring zinc to antioxidant zincdependent enzymes and proteins. ${ }^{27,28}$

Compared to control, the expression of both p53 and PARP-1, also named "guardians of the genome" due to their function in maintaining genome integrity, could represent a complementary and additional way by which testes cells may face the negative effects of ROS accumulation. ${ }^{29}$

PARP-1 is a zinc-finger DNA-binding enzyme able to recognize DNA damages, DNA nicks and double stranded breaks, and once activated catalyzes the synthesis of branched poly(ADPribose) polymers that stabilize the surrounding chromatin and converts DNA damages into intracellular signals which activate DNA repair programs. ${ }^{29}$ In this regard, a similar function of PARP could be also assumed in rat testes' cells in response to SMG-induced oxidative stress. By the same token, the up-regulation in p53 expression, observed in the present work, is probably due to its pivotal role in regulating cell response to multiple stress signals, including the oxidative stress.

Moreover, we observed mitochondrial dysregulation in treated cells. Similar effects, although occurring in different species, suggests that mitochondria changes could be a stress adaptive response to ensure cell energy. Considering that mitochondria are the major targets of ROS negative effects, an increase of free radicals and other reactive oxygen species is able to induce a mitochondrial membrane permeabilization, a process that could leads to mitopsosis and mitophagy, in order to eliminate dysfunctional mitochondria and protect cells. ${ }^{30}$

Taking the current data together, it seems that short-term SMG exposure induced morphofunctional alteration and an increase in oxidative stress response in rat primary testis culture, in agreement with results obtained in other works. ${ }^{19,20,28}$

\section{Conclusions}

In this study, we validated our hypothesis that short-term SMG induces oxidative cellular stress in rat testes primary culture cells, as demonstrated by the response of the antioxidant system and impaired mitochondrial function. We hypothesized that the significant expression level increase of oxidative stress-induced gene, as GST and MT1, PARP and p53 could be related to an increase in ROS production. ROS level the observed morpho-functional, in cytoskeleton, SHBG/ABP production, cell viability, could have a significant inhibitory effect on germ cell differentiation which may result in male infertility.

Our data ought to provide important insight in this topic concerning the effect of short-term SMG exposure, but also in $1 \mathrm{~g}$ condition, because of male infertility is also a social concern, considering the increase of life expectancy. These findings could lead the way to evaluate steroidogenesis, spermatogenesis and cell senescence via oxidant stress mechanisms induced by microgravity, performing further investigation during simulated or actual spaceflights.

\section{References}

1. Mishra B, Luderer U. Reproductive hazards of space travel in women and men. Nat Rev Endocrinol 2019;15:713-30.

2. Tou J, Ronca A, Grindeland R, Wade C. Models to study gravitational biology of mammalian reproduction. Biol Reprod 2002;67:1681-87.

3. Zhu H, Wang H, Liu Z. Effects of real and simulated weightlessness on the cardiac and peripheral vascular functions of humans: a review. Int J Occup Med Environ Health 2015;28:793-802.

4. Tanaka K, Nishimura N, Kawai Y. Adaptation to microgravity deconditioning and countermeasures. J Physiol Sci 2017;67:271-81. 
5. Sominsky L, Hodgson DM, McLaughlin EA, et al. Linking stress and infertility: a novel role for ghrelin. Endocr Rev 2017;38:432-67.

6. Nargund VH. Effects of psychological stress on male fertility. Nat Rev Urol 2015;12:373-82.

7. Herranz R, Anken R, Boonstra J, et al. Ground-based facilities for simulation of microgravity: organism-specific recommendations for their use and recommended terminology. Astrobiology 2013;13:1-17.

8. Grindeland RE, Popova IA, Vasques M, Arnaud SB. Cosmos 1887 mission overview: effects of microgravity on rat body and adrenal weights and plasma constituents. FASEB J 1990;4:105-9.

9. Hadley JA, Hall JC, O'Brien A, Ball R. Effects of a simulated microgravity model on cell structure and function in rat testis and epididymis. J Appl Physiol 1992;72:748-59

10. Vasques M, Lang C, Grindeland RE, et al. Comparison of hyper- and microgravity on rat muscle organ weights and selected plasma constituents. Aerosp Med Hum Perform 1998;69:2-8.

11. Strollo F, Barger L, Fuller C. Testosterone urinary excretion rate increases during hypergravity in male monkeys. J Gravit Physiol 2000; 7:181-82.

12. Masini MA, Albi E, Barmo C, et al. Impact of long-term exposure to space environment on adult mammalian organisms: a study on mouse thyroid and testis. Plos one 2012;7:e35418.

13. Smith SM, Heer M, Wang Z, et al. Long-duration space flight and bed rest effect on testosterone and other steroids. J Clin Endocrinol Metab 2012;97:270-78.

14. Uva BM, Masini MA, Sturla M, et al. Clinorotation-induced weightlessness influences the cytoskeleton of glial cells in culture. Brain Res 2002;934:132-39.

15. Sciola L, Cogoli-Greuter M, Cogoli A, et al. Influence of microgravity on mitogen binding and cytoskeleton in Jurkat cells. Adv Space Res 1999;24:801-5.

16. Infanger M, Kossmehl P, Shakibaei M, et al. Simulated weightlessness changes the cytoskeleton and extracellular matrix proteins in papillary thyroid carcinoma cells. Cell Tissue Res 2006;324:267-77.

17. Tauber S, Hauschild S, Paulsen K, et al. Signal transduction in primary human $\mathrm{T}$ lymphocytes in altered gravity during para- bolic flight and clinostat experiments. Cell Physiol Biochem 2015;35:1034-51.

18. Gmunder FH, Kiess M, Sonnefeld G, et al. A ground-based model to study the effects of weightlessness in lymphocytes. Biol Cell 1990;70:33-38.

19. Uva BM, Strollo F, Ricci F, et al. Effect of conditions of three dimensional clinostating on testicular cell machinery. Acta astronautica 2007;60:391-96.

20. Masini M, Prato P. Effects of a simulated microgravity model on cell structure and function in mouse testis. J Biol Res 2010;83:29-32.

21. Aleshcheva G, Sahana J, Ma X, et al. Changes in morphology, gene expression and protein content in chondrocytes cultured on a random positioning machine. Plos One 2013;8:e79057.

22. Van Borst AG, van Loon JJWA. Technology and development for the random positioning machine, rpm. Micorgravity Sci Technol 2009;21:287.

23. Coons AH, Leduc EH, Connolly JM. Studies on antibody I A method for the histochemical demonstration of specific antibody and its application to a study of the hyperimmune rabbit. J Exper Med 1955;102:49-59.

24. Chomczynski P, Sacchi N. Single-step method of RNA isolation by acid guanidinium thiocyanate-phenol-chloroform extraction. Anal Biochem 1978;162:156-59.

25. Pfaffl MW, Horgan GW, Dempfle L. Relative expression software tool (REST) for group-wise comparison and statistical analysis of relative expression results in real-time PCR. Nucleic Acids Res 2002;30-6.

26. Vandesompele J, De Preter K, Pattyn F, et al. Accurate normalization of real-time quantitative RT-PCR data by geometric averaging of multiple internal control. Genome Biol 2002;3:1-12.

27. Morabito C, Guarnieri S, Catizone A, et al. Transient increases in intracellular calcium and reactive oxygen species levels in TCam-2 cells exposed to microgravity. Sci Rep 2017; 7:148-56.

28. Aitken RJ, Roman SD. Antioxidant systems and oxidative stress in the testes. Oxid Med Cell Longev 2008;1:15-24.

29. Elkholi R, Chipuk JE. How do I kill thee? Let me count the ways: p53 regulates PARP-1 dependent necrosis. Bio Essays 2014;36:46-51.

30. Venditti P, Di Meo S. The role of reactive oxygen species in the life cycle of the mitochondrion. Int J Mol Sci 2020;21:21-73. 